Article

Fl. Medit. 31 (Special Issue): 95-104

https://doi.org/10.7320/FlMedit31SI.095

Version of Record published online on 16 July 2021

\author{
R. Venanzoni, S. Praleskouskaya \& G. Ciaschetti
}

\title{
Contribution to the Syntaxonomy of Rare Tall Sedge Community in Central Apennine (Umbria-Italy): I. Caricetum buxbaumii
}

\begin{abstract}
Venanzoni, R., Praleskouskaya, S. \& Ciaschetti, G.: Contribution to the Syntaxonomy of Rare Tall Sedge Community in Central Apennine (Umbria-Italy): I. Caricetum buxbaumii. F1. Medit. 31 (Special Issue): 95-104. 2021. - ISSN: 1120-4052 printed, 2240-4538 online.

This contribution describes a new plant association of marshy meadows, named Deschampsio cespitosae-Caricetum buxbaumii ass. nova hoc loco, occurring in the karstic basins of Sibillini Mountains (Pian Grande, Pian Piccolo and Savelli-Castel S. Maria, Umbria Region) between 1000-1350 m above sea level. The new association is distinguished from Ranunculo-Caricetum buxbaumii Pedrotti 2016 and Galio palustris-Caricetum buxbaumii Ciaschetti \& al. 2020 for its floristic and ecological features. After the comparison with the original tables of Issler's Caricetum buxbaumii and Ritter-Studnicka's Valeriano-Caricetum buxbaumii, all Apennine associations belong to Caricion gracilis alliance, and are southern synvicariants of the Caricetum buxbaumii Issler 1933 of Central Europe.
\end{abstract}

Key words: Carex buxbaumii communities, Caricion gracilis, Apennine Karstic plains, Central Italy.

\section{Introduction}

The vegetation of the Magnocaricetalia elatae Pignatti 1953 Order has been the subject in recent years of numerous studies aimed at finding common traits for classification at the European level (Mucina \& al. 2016, Landucci \& al. 2020) or to look for distinctive elements that characterize these plant communities distributed on a continental scale at the local level (Gigante \& Venanzoni 2000; Pedrotti 2015, 2016b; Ciaschetti \& al. 2020, Landucci \& al. 2013; Lastrucci \& al. 2012, 2014). Swamps, with large sedges, have now almost all disappeared or deeply transformed throughout the Italian plains and hilly territory due to reclamations and anthropic pressure. In this context, the karstic plains, which range along the Apennine ridge at an altitude of 900-1300 $\mathrm{m}$ from the Umbro-Marchigiano Apennines, up to the Abruzzo and Molise Regions (Pedrotti 1976), represent a very important subsystem of the Apennine ecoregional Province (Blasi \& al. 2018) and an exceptional reservoir of biodiversity hosting almost all the species that characterize the vegetation of the Magnocaricetetalia (Ciaschetti \& al. 2020). 
Carex buxbaumii Wahlenb. is an amphiatlantic boreal species with a still unknown range of distribution (Segal \& Westhoff 1959). It is a rare and threatened species in Europe (Sotek 2006; e.g. Grulich 2012; Bernátová \& al. 2018). It is rare also in Italy (Pedrotti 1971) and included in the Read Lists of The Italian Flora as Endangered (Rossi \& al. 2013); The EEC Habitat Directive 92/43 does not include any rare tall sedge communities. Only Poland and some other Nordic countries (Estonia, Latvia, etc.), where Caricetum buxbaumi is attributed to Caricion davallianae, can protect this association by including it in Habitat $7210^{*}$ "Calcareous fens with Cladium mariscus and species of the Caricion davallianae" and in Habitat 7230 “Alkaline fens" (Wolejko \& al. 2005; Bacieczko \& Kaszycka 2015; Stanko \& al. 2018) though it has been stated for this association a severe reduction (Wolejko \& al. 2005). The aim of this paper is to present a comparison of the communities of Caricetum buxbaumii of the Apennines with those analyzed in the recent European literature (e.g. Landucci \& al. 2020).

\section{Materials and methods}

We have considered 34 phytosociological relevés (according to Braun-Blanquet 1964) from Umbria and Abruzzo Regions (Fig. 1): 10 unpublished (made in the Sibillini Mountains) and 24 taken from the literature (Gigante \& Venanzoni 2005; Pedrotti 2015 and Ciaschetti \& al. 2020) were rearranged in order to compare the classification with recent European literature (e.g. Landucci \& al. 2020). Floristic nomenclature follows Pignatti (2017-2019) and Bartolucci \& al. (2018) for vascular plants; Aleffi \& al. (2020) for mosses.

We have applied cluster analysis by complete linkage using similarity ratio as resemblance function by the Matedit software (Burba \& al. 1992, downloadable at www.vegitaly.it). Relevés are stored in the free botanical database http:/www.anachive.it (Landucci \& al. 2012; Gigante \& al. 2012; Lucarini \& al. 2015).

\section{Results}

Deschampsio caespitosae-Caricetum buxbaumii ass. nova. hoc. loco (Table 1, holotypus: rel. 5 of Table 1)

\section{Floristic analysis}

The new association is characterized by a general depletion of species of the Magnocaricetalia Order (evidenced for all communities also in Landucci \& al. 2013, 2020; Venanzoni \& al. 2018; Ciaschetti \& al. 2020) and the presence of transitional meadows species such as Deschampsia caespitosa, Ranunculus repens and Potentilla reptans (typical and diagnostic species are highlighted in Table 1). The comparison with the relevés of central Italy (Table 1) shows a clear separation between that of the proposed association and those described as Ranunculo pedrotti-Caricetum buxbaumii Pedrotti 2015 and Galio palustris-Caricetum buxbaumii Ciaschetti \& al. 2020; the cluster analysis (Table 1) confirms a sharp classification that not made possible to include all relevés in one association. 


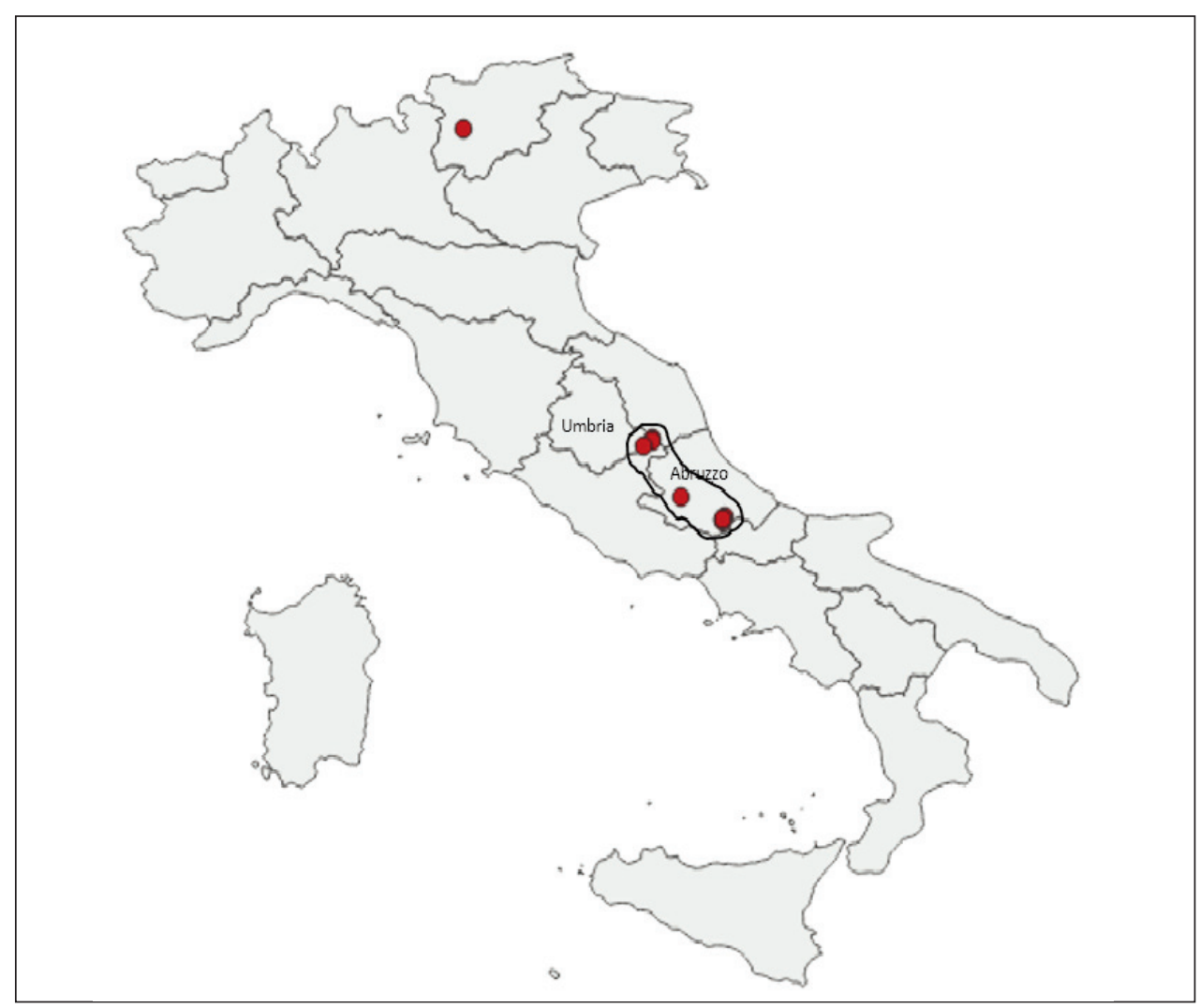

Fig. 1. Distribution of Carex buxbaumii in Italy (source www.anarchive.it) and study area.

Lastly, the relevés have been compared with the original tables of Issler's Caricetum buxbaumii and Ritter-Studnicka's Valeriano-Caricetum buxbaumi (results are shown in the Electronic Supplemetary File 1). Both Caricetum buxbaumii and Valeriano-Caricetum buxbaumii are characterized by species of Molinietalia cerulae and Caricetalia davallianae due to vegetational catenal contact with peaty vegetation that does not happen in Central Italy where we found only catenal contacts with transitional meadows of Potentilletalia anserinae and Trifolio-Hordeetalia secalini. Same conclusion may be assumed also by comparing Table 2 with Table 1 in Segal \& Westhof (1959); in fact, these authors define the Caricetum buxbaumii as a transitional association between the Molinietalia and the Schuchzerio-Caricetea fuscae.

\section{Synecology}

Deschampsio-Caricetum buxbaumii develops in stations with high water excursion and summer water stress conditions that occur in the karst bassin of Savelli-Castel Santa Maria (where it forms extensive populations, Fig. 2) and in the Pian Piccolo of Castelluccio. 
98 Venanzoni \& al.: Contribution to the Syntaxonomy of Rare Tall Sedge Community ...

Table 1. Dendrogram \& Classification of the 34 relevés: Legenda: PP (Pian Piccolo di Norcia - PG), PG (Pian Grande di Norcia - PG), AB (Abruzzo), SM (Savelli-Castel S. Maria - PG)

Source of data: Rel. 2-7, 9-12 (original data), rel. 1-8, 17, 28-30 (Gigante \& Venanzoni 2005), rel. 17-27, 31-34 (Pedrotti 2015); rel. 13-16 (Ciaschetti \& al. 2020).

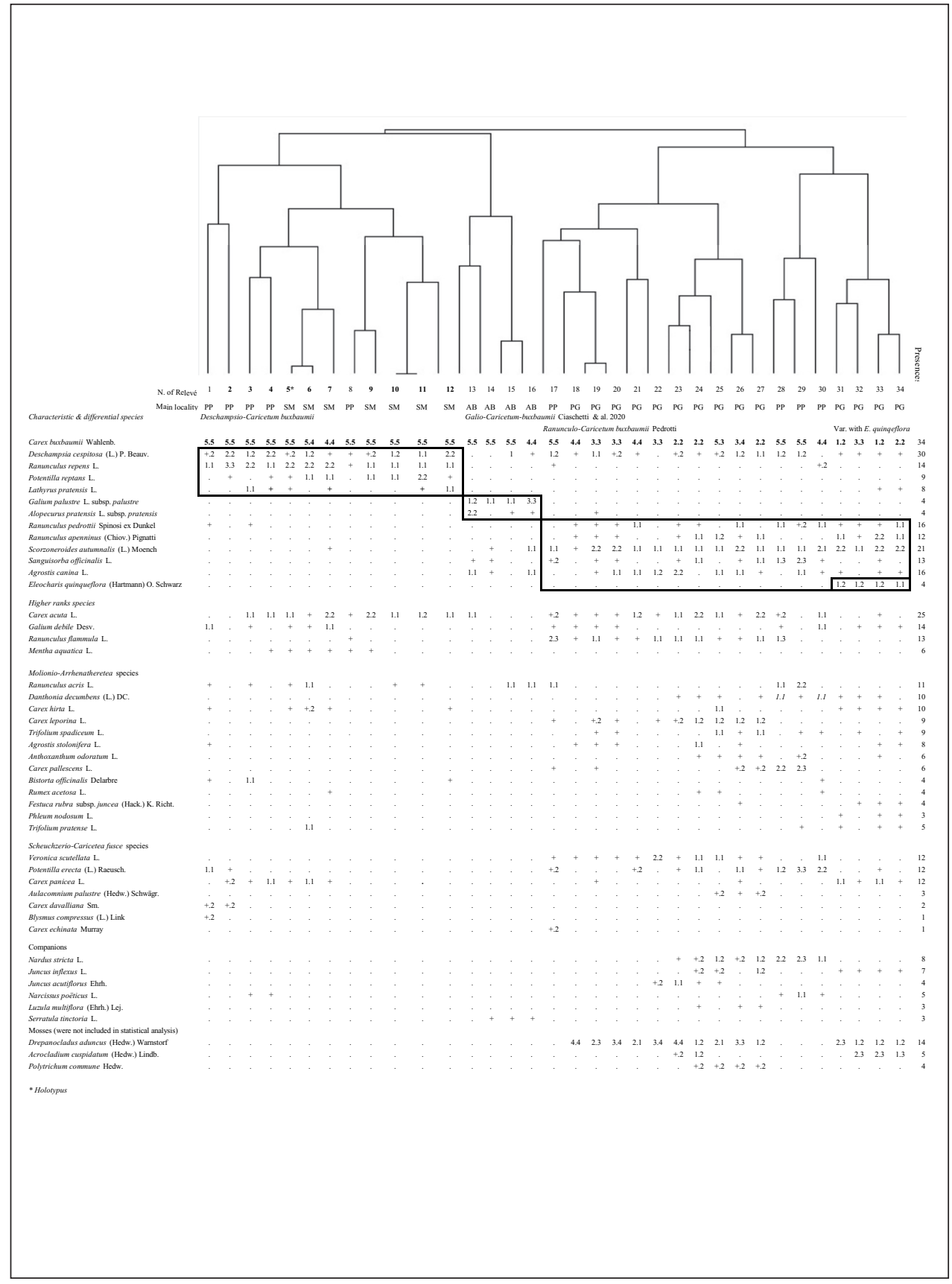




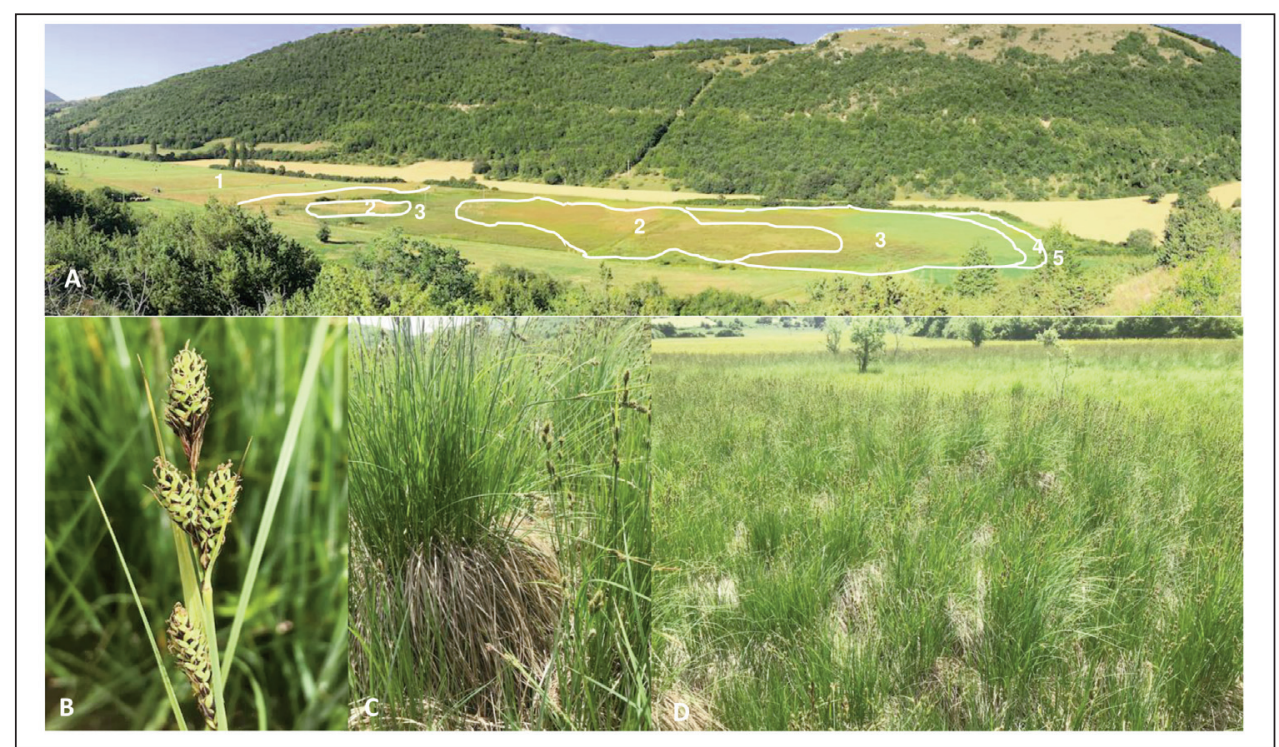

Fig. 2. Schematic representation of the vegetation in the study area (Savelli/S. Maria Karstic plain). A: general view with 1 Trifolio-Hordeetalia, 2 Deschampsio-Caricetum buxbaumii, 3 Caricetum gracilis. 4 Caricetum vesicariae, 4 Sinkhole. B: Carex buxbaumii. C: life forms of C. buxbaumi, D: DeschampsioCaricetum buxbaumii.

\section{Synchorology}

Deschampsio caespitosae-Caricetum buxbumii is present in the Karstic bassin of Savelli-Castel Santa Maria and Piani di Castelluccio, Ranunculo pedrotti-Caricetum buxbaumii only in the Piani di Castelluccio (Pedrotti 2015) and, finally, Galio palustris-Caricetum buxbaumii in the Major Highlands of Abruzzo (Ciaschetti \& al 2020).

\section{Final Remarks}

The Carex buxbaumii populations of Central Italy constitute a distinct and isolated post glacial relict nucleus with distinct floristic, ecological, and historical characteristics that allow us to include them in the Caricion gracilis Alliance. The Caricetum buxbaumii Issler 1932 can be considered present only in the southern slope of the Italian Alps (where the species is reported) in contact with the peaty meadows vegetation of Molinietalia and Scheucherio-Caricetea fuscae. On the contrary, the Carex buxbaumii communities of the Apennine karstic plains, in catenal contact with the transition meadows of Deschampsion caespitosae and Potentillion anserinae, attributable to the habitat 3180 * "Turloughs" not currently present in the Italian Interpretation Manual (http://vnr.unipg.it/habitat), are included into the Caricion gracilis Alliance. The present research highlights that the Habitat Directive 92/43 does not include any Habitat with the vegetation of the Phragmiti-Magnocaricetea 
100 Venanzoni \& al.: Contribution to the Syntaxonomy of Rare Tall Sedge Community ...

Class and therefore there are no tools of protection neither at the National nor at the European level for the very rare associations Caricetum buxbaumii - treated in this work - and others such as Caricetum vulpinae and Caricetum distichae.

\section{Syntaxomic scheme}

SCHEUCHZERIO PALUSTRIS-CARICETEA NIGRAE NOM. MUT. PROPOS. EX STEINER 1992

\section{Caricetalia davallianae Br.-Bl 1949}

Caricion davallianae

Valeriano simplicifoliae-Caricetum buxbaumii Ritter-Studnicka 1972

Phragmiti-Magnocaricetea elatae KLiKa in KliKa \& NovÁK 1941

Magnocaricetalia elatae Pignatti 1953

Caricion elatae Koch 1926

Caricetum buxbaumii Issler 1932

Caricion gracilis Gèhu 1961

Ranunculo pedrotti-Caricetum buxbaumii Pedrotti 2016

Galio palustris-Caricetum buxbaumii Ciaschetti, Pirone \& Venanzoni 2020

Deschampsio cespitosae-Caricetum buxbaumii Venanzoni, Praleskouskaya \& Ciaschetti 2021

\section{References}

Aleffi, M., Tacchi, R. \& Poponessi, S. 2020: New Checklist of the Bryophytes of Italy. - Cryptog. Bryol. 41(13): 147-195. https://doi.org/10.5252/cryptogamie-bryologie2020v41a13.

Bacieczko, W. \& Kaszycka, E. 2015: The conservation requirements of rare and threatened vascular plants of Natura 2000 habitats of the Dolina Płoni i Jezioro Miedwie (Płonia valley and Miedwie lake) special area of conservation. - Folia Pomeranae Univ. Technol. Stetinensis 316(33): 15-32.

Balatova-Tulackova, E. 1972: Flachmoorwiesen im mittleren und unteren Opava-Tal (Schlesien). Academia-Verlag Tschechoslow, Akad. Wissen. - Prague.

Bartolucci, F., Peruzzi, L., Galasso, G., Albano, A., Alessandrini, A., Ardenghi, N. M. G., Astuti, G., Bacchetta, G., Ballelli, S., Banfi, E., Barberis, G., Bernardo, L., Bouvet, D., Bovio, M., Cecchi, L., Di Pietro, R., Domina, G., Fascetti, S., Fenu, G., Festi, F., Foggi, B., Gallo, L., Gottschlich, G., Gubellini, L., Iamonico, D., Iberite, M., Jiménez-Mejías, P., Lattanzi, E., Marchetti, D., Martinetto, E., Masin, R. R., Medagli, P., Passalacqua, N. G., Peccenini, S., Pennesi, R., Pierini, B., Poldini, L., Prosser, F., Raimondo, F. M., Roma-Marzio, F., Rosati, L., Santangelo, A., Scoppola, A., Scortegagna, S., Selvaggi, A., Selvi, F., Soldano, A., Stinca, A., Wagensommer, R. P., Wilhalm, T. \& Conti, F. 2018: An updated checklist of the vascular flora native to Italy. - Pl. Biosyst. 152(2): 179-303. https://doi.org/10.1080/11263504.2017.1419996

Bernátová, D., Kliment, J., Topercer, J. 2018: Buxbaum's sedge - a hot candidate for extinction in Slovakia. - Bull. Slov. Bot. Spoločn. 40(2): 171-180.

Blasi, C., Capotorti, G., Copiz, R. \& Mollo, B. 2018: A first revision of the Italian Ecoregion map. - Pl. Biosyst. 152(6): 1201-1204. https://doi.org/10.1080/11263504.2018.1492996

Braun-Blanquet, J. 1964: Pflanzensoziologie - Grundzuge der Vegetationkunde. - Wien, New York.

Burba N., De Paoli, D., Feoli, E., Malaroda, M., Oriolo, G. \& Zuccarello, V. 1992: Un sistema informativo per la vegetazione. Calcolo degli indici ecologici. - Coll. Quad. C.E.T.A., 2. - Gorizia. 
Ciaschetti, G., Pirone, G. \& Venanzoni, R. 2020: Sedge Vegetation of the "Major Highlands of Abruzzo" (Central Italy): Updated Knowledge After New Discoveries. - Pl. Biosyst. 155(3): 647-662. https://doi.org/10.1080/11263504.2020.1801876

Conti, F., Pedrotti, F. \& Pirone, G. 1990: Su alcune piante notevoli rinvenute in Abruzzo, Molise e Basilicata. - Arch. Bot. Ital. 66(3/4): 1-15.

Cortini Pedrotti, C., Orsomando, E., Pedrotti, F. \& Sanesi, G. 1973: La vegetazione e i suoli del Pian Grande di Castelluccio di Norcia (Appennino centrale). - Atti Ist. Bot. Lab. Critt. Univ. Pavia 9: 155-249.

Dunkel, F. G. 2011: The Ranunculus auricomus L. complex (Ranunculaceae) in Central and Southern Italy, with addition to north Italian taxa. - Webbia 66(2): 165-193. https://doi.org/10.1080/00837792.2011.10670895

Ferrez, Y. 2005: Connaissance de la flore rare ou menacée de Franche-Comté, Carex buxbaumii Wahlenb. - Besançon.

Gallandat, J.-D. 1982: Prairies marécageuses du Haut-Jura (Molinietalia, Scheuchzerio-Caricetea fuscae et Phragmitetea). - Matér. Levé Géobot. Suisse 58: 1-180.

Gigante, D. \& Venanzoni, R. 2000: Contributo alla conoscenza della vegetazione degli ambienti umidi dell'Umbria (Italia). - Fitosociologia 37(2): 13-63.

— \& - 2005: Habitat umidi di rilevante interesse botanico e vegetazionale nel Pian Piccolo di Castelluccio (PG). - In: Guida all'escursione della Società Italiana di Scienza della Vegetazione.

-, Acosta, A. T. R., Agrillo, E., Attorre, F., Cambria, V. E., Casavecchia, S., Chiarucci, A., Del Vico, E., De Sanctis, M., Facioni, L., Geri, F., Guarino, R., Landi, S., Landucci, F., Lucarini, D., Panfili, E., Pesaresi, S., Prisco, I., Rosati, L., Spada, F. \& Venanzoni, R. 2012: VegItaly: Technical features, crucial issues and some solutions. - Pl. Sociol. 49(2): 71-79. https://doi.org/10.7338/pls2012492/05

Grulich, V. 2012: Red List of vascular plants of the Czech Republic: 3rd edition. - Preslia 84: 631-645.

Issler, E. 1933: Les prairies non fumées du Ried Ello-rhénan et le Mesobrometum du Haut-Rhin. - Bull. Soc. Hist. Nat. Colmar 23: 43-129.

Jutrzenka-Trzebiatowski, A. \& Szarejko, T. 2001: Zespol Caricetum buxbaumii w Wigierskim Parku Narodowym. - Fragm. Flor. Geobot. Polonica 8: 149-171.

Keller, L. 1969-1970: Étude des groupements végétaux de la réserve de Cheyres. - Bull. Soc. Fribourgeoise Sc. Nat. Neuchâtel 59: 47-59.

Klötzli, F. 1969: Die Grundwasserbeziehungen der Streu- und Moorwiesen in nördlichen Schweizer Mittelland. - Beitr. Geobot. Landesaufnahme Schweiz 52: 1-296.

— 1973: Übersicht über die walfreien Nassstandorte der Schweiz. Veröff. Geobot. - Inst. Eidg. Techn. Hochschule, Stift. Rübel, Zürich 51: 15-39.

Korneck, D. 1962: Die Pfeifengraswiesen und ihre wichtigsten Kontaktgesellschaften in der nördlichen Oberrheinebene und in Schweinfurter Trockengebiet I. - Beitr. Naturk. Forsch. SW-Deutschl. 21(1): 55-77.

Korneck, D. 1963: Die Pfeifengraswiesen und ihre wichtigsten Kontaktgesellschaften in der nördlichen Oberrheinebene und in Schweinfurter Trockengebiet III. - Beitr. Naturk. Forsch. SW-Deutschl. 22(1): 19-44.

Landucci, F., Acosta, A. T. R., Agrillo, E., Attorre, F., Biondi, E., Cambria, V. M., Chiarucci, A., Del Vico, E., De Sanctis, M., Facioni, L., Geri, F., Gigante, D., Guarino, R., Landi, S., Lucarini, D., Panfili, E., Pesaresi, S., Prisco, I., Rosati, L., Spada, F. \& Venanzoni, R. 2012: VegItaly: The Italian collaborative project for a national vegetation database. - P1. Biosyst. 146(4): 756-763. https://doi.org/10.1080/11263504.2012.740093

—, Gigante, D., Venanzoni, R. \& Chytrý, M. 2013: Wetland vegetation of the class Phragmito-Magnocaricetea in Central Italy. - Phytocoenologia 43(1-2): 67-100, https://doi.org/10.1127/0340269X/2013/0043- 0545 
102 Venanzoni \& al.: Contribution to the Syntaxonomy of Rare Tall Sedge Community ...

—, Sumberová, K., Tichý, L., Hennekens, S., Aunina, L., BițăNicolae, C., Borsukevych, L., Bobrov, A., Carni A., De Bie, E., Golub V., Hrivnák, R., Iemelianova, S., Jandt, U., Jansen, F., Kącki, Z., Lájer, K., Papastergiadou, E., Silc, U., Sinkevičene; Z., Stančŕc, Z., Stepanovič, J., Teteryuk, B., Tzonev, R., Venanzoni, R., Zelnik, I. \& Chytrý, M. 2020: Classification of the European marsh vegetation (Phragmito-Magnocaricetea) to the association level. - Appl. Veg. Sci. 23: 297-316, https://doi.org/10.1111/avsc.12484

Lastrucci, L., Landucci, F., Gonnelli, V., Barocco, R., Foggi, B. \& Venanzoni, R. 2012: The vegetation of the upper and middle River Tiber (Central Italy). - Pl. Sociol. 49(2): 29-48.

-, Bonari, G., Angiolini, C., Casini, F., Giallonardo, T., Gigante, D., Landi, M., Landucci, F., Venanzoni, R. \& Viciani, D. 2014: Vegetation of Lakes Chiusi and Montepulciano (Siena, Central Italy). Plant Sociology 51: 29-55. https://doi.org/10.7338/pls2014512/03

Lucarini, D., Gigante, D., Landucci, F., Panfili, E. \& Venanzoni, R. 2015: The anArchive taxonomic Checklist for Italian botanical data banking and vegetation analysis: Theoretical basis and advantages. - Pl. Biosyst. 149(6): 958-965. https://doi.org/10.1080/11263504.2014.984010

Mucina, L., Bültmann, H., Dierßen, K., Theurillat, J.-P., Raus, T., Čarni, A., Šumberová, K., Willner, W., Dengler, J., García, R. G., Chytrý, M., Hàjek, M., Di Pietro, R., Iakushenko, D., Pallas, J., Daniëls, F. J. A., Bergmeier, E., Guerra, A. S., Ermakov, N., Valachovič, M., Schaminée, J. H. J., Lysenko, T., Didukh, Y. P., Pignatti, S., Rodwell, J. S., Capelo, J., Weber, H. E., Solomeshch, A., Dimopoulos, P., Aguiar, C., Hennekens, S. M. \& Tichý, L. 2016. Vegetation of Europe: Hierarchical floristic classification system of vascular plant bryophyte lichen and algal communities. - Appl. Veg. Sci. 19(S1): 3-264.

Oberdorfer, E. 1957: Süddeutsche Pflanzengesellschaften. - Jena.

- 1983: Pflanzensoziologische Exkursionsflora. - Stuttgart.

Pedrotti, F. 1971: Carex buxbaumii, relitto glaciale: prima segnalazione per l'Italia peninsulare. - Arch. Bot. Biogeogr. Ital. 15(1): 21-28.

- 1976: Les prairies permanentes humides de l'Apennin central: phytosociologie et cartographie. - Coll. Phytosoc. 5: 181-187.

- 1982: La végétation du Pian Grande. - Pp. 347-360 in: Guide-Itinéraire Excursion Internat. Phytosociologie Italie centrale (2-11 juillet 1982). - Camerino.

- 2015: A new plant association of Caricion gracilis alliance of the Central Apennines (Italy). - Contrib. Bot. 50: 137-152.

- 2016a: ERRATUM. A new plant association of Caricion gracilis alliance of the central Apennines (Italy). - Contrib. Bot. 51: 155.

- 2016b: The Caricetum distichae Association in Italy. - Pp. 357-362 in: Vegetation structure and function at multiple spatial, temporal and conceptual scales. Geobotany studies - New York.

— \& Sanesi, G. 1969: Resoconto delle escursioni sull'Appennino umbro-marchigiano (25-27 giugno 1968). - Mitt. ostalp.-din. pflanzensoz. Arbeitsgem. Camerino 9: 365-388.

-, Aleffi, M. \& Cortini Pedrotti, C. 2004: La vegetazione attuale dei depositi quaternari dell'Umbria e delle Marche (Italia centrale). - Coll. Phytosoc. 28: 1073-1084.

Philippi, G. 1960: Zur Gliederung der Pfeifengraswiesen im südlichen und mittleren Oberrheingebiet. Beitr. Naturk. Forsch. Südw.-Dtld. 19: 138-187.

- 1971: Beiträge zur Flora der nordbadischen Rheinebene und der angrenzenden Gebiete. - Beitr. Naturk. Forsch. Südw.-Dtld. 30: 9-47.

Pignatti, S., Guarino, R. \& La Rosa, M. 2017-19. Flora d'Italia, 1-4, 2 ed, \& Flora Digitale. - Milano.

Ritter-Studnicka, H. 1972: Neue Pflanzengesellschaften aus den Karstfeldern Bosniens und der Hercegovina. - Bot. Jahrb. Syst. Stuttgart 92: 108-154.

Rossi, G., Montagnani, C., Gargano, D., Peruzzi, L., Abeli, T., Ravera, S., Cogoni, A., Fenu, G., Magrini, S., Gennai, M., Foggi, B., Wagensommer, R. P., Venturella, G., Blasi, C., 
Raimondo, F. M. \& Orsenigo, S. (eds) 2013: Lista Rossa della Flora Italiana. 1. Policy Species e altre specie minacciate. - Roma.

Salmina, L. 2005: New fen communities in Latvia. - Latvijas Univ. Raksti 685: 96-111.

- 2006: Limnogenous, mire vegetation of Latvia. - Summary of the PhD thesis, University of Latvia Riga.

Segal, S., Westhoff, V. \& Dijk, J. (van) 1959: Die Vegetationskundliche Stellung von Carex buxbaumii Wahlenb. in Europa, besonders in den Niederlanden. - Acta Bot. Neerl. 8: 304-329.

Sotek, Z. 2006: The distribution of Carex buxbaumii Wahlenb. in Poland. - Acta Societatis Botanicorum Poloniae 75(4): 293-296.

Stańko, R., Wołejko, L. \& Pawlaczyk, P. (eds) 2018: A Guidebook on Good Practices of alkaline fen conservation. - Świebodzin.

Tomaszevic, H. 1979: Roslinnosc wodna i szuwarowa Polski. - Warsaw.

Venanzoni, R., Properzi, A., Bricchi, E., Landucci, F. \& Gigante D. 2018: The Magnocaricetalia Pignatti 1953 (Phragmito- Magnocaricetea Klika in Klika et Novák 1941) Plant Communities of Italy. Pp. 135-173 in: Pedrotti F. (ed.), Climate Gradients and Biodiversity in Mountains of Italy. Geobotany Studies, Berlin. https://doi.org/10.1007/978-3-319- 67967-9_8

Wolejko, I., Herbichowa, M. \& Potocka, J. 2005: Typological differentiation and status of Natura 2000 mire habitats in Poland. - Stapfia 85, zugleich Kataloge der Ö. Landesmuseen Neue Serie 35: 175-219.

Addresses of the authors:

Roberto Venanzoni ${ }^{1}$, Safiya Praleskouskaya ${ }^{1} \&$ Giampiero Ciaschetti ${ }^{2}$

${ }^{1}$ Dept of Chemistry, Biology and Biotechnologies, University of Perugia, Polo Didattico V. del Giochetto 6, 06122 Perugia - Italy. E-mail: roberto.venanzoni@unipg.it

${ }^{2}$ Majella National Park, via Badia 28, 67039 Sulmona (AQ) - Italy. 
104 Venanzoni \& al.: Contribution to the Syntaxonomy of Rare Tall Sedge Community ...

\section{Annex 1 - Sporadic species of Tab. 1 [specie, value (relevés)].}

Ophioglossum vulgatum L., 1,1 (21), +(26); Schedonorus arundinaceus (Schreb.) Dumort. + (5), +.2(6); Galium mollugo L. 1.1 (11), 2.2 (12); Trifolium repens L. 1.1 (6), +(34); Alopecurus rendlei Eig. 2.2(15), +(16); Briza media L. +(1), +.2(2); Taraxacum officinale Weber (aggr.), +(12), +(26); Cerastium holosteoides Fr., +(5), +(6); Lathyrus pannonicus (Jacq.) Garcke +(15), +(16); Bellevalia romana (L.) Sweet $+(14),+(16)$; Vicia cracca L. + (27), + (34); Rhinanthus minor L. + (29) + (31); Carex vulpina L. + (34); Equisetum palustre L. + (20); Trifolium fragiferum L. 1.1 (6); Cardamine amporitana Sennen et Pau + (1); Rubus caesius L. + (5); Carex disticha Huds. + (13); Cruciata pedemontana (Bellardi) Ehrend. + 2; Galium verum L. +.2 (30); Oenanthe fistulosa L. + (14); Veronica chamaedrys L. + (1) + (2); Euphrasia stricta J. P. Wolff ex J.F. Lehm. + (21); Cynosurus cristatus L. + (31); Centaurea jacea L. + (33); Dicranum bonjeanii De Not. +.2(4); Campylium polygamum (Schimp.) CEO Jensen + (31); Luzula spicata (L.) DC. + (29); Juncus articulatus L. +.2 (1); Juncus conglomeratus L. +.2 (29); Ornithogalum gussonei Ten. + (29); Rumex acetosella L. + (30).

\section{Annex 2 - Locality and data of unpublish relevés.}

\begin{tabular}{|l|c|c|c|c|c|c|c|c|c|c|}
\hline $\begin{array}{l}\text { Number of relevés in } \\
\text { this paper }\end{array}$ & 2 & 3 & 4 & 5 & 6 & 7 & 9 & 10 & 11 & 12 \\
\hline Original \# of relevé & PPSN3 & PPSN2 & PPSN1 & A7 & Al & 19 & A6 & A5 & A3 & A2 \\
\hline Locality & Pian Piccolo & $\begin{array}{c}\text { Pian } \\
\text { Piccolo }\end{array}$ & Pian Piccolo & $\begin{array}{c}\text { Savelli- } \\
\text { CSM }\end{array}$ & $\begin{array}{c}\text { Savelli- } \\
\text { CSM }\end{array}$ & $\begin{array}{c}\text { Savelli- } \\
\text { CSM }\end{array}$ & $\begin{array}{c}\text { Savelli- } \\
\text { CSM }\end{array}$ & $\begin{array}{c}\text { Savelli- } \\
\text { CSM }\end{array}$ & $\begin{array}{c}\text { Savelli-CSM } \\
\text { Savelli-CSM }\end{array}$ \\
\hline Date (DD/MM/YY) & $16 / 06 / 18$ & $16 / 07 / 20$ & $16 / 07 / 18$ & $09 / 06 / 16$ & $10 / 06 / 16$ & $22 / 07 / 18$ & $09 / 06 / 16$ & $09 / 06 / 16$ & $09 / 06 / 16$ & $09 / 06 / 16$ \\
\hline Latitude N & 42.769994 & 42.769994 & 42.769994 & 42.715220 & 42.715220 & 42.715220 & 42.715220 & 42.715220 & 42.715220 & 42.715220 \\
\hline Longitude E & 13.207399 & 13.207399 & 13.207399 & 13.106141 & 13.106141 & 13.106141 & 13.106141 & 13.106141 & 13.106141 & 13.106141 \\
\hline Altitude (m a.s.l.) & 1332 & 1332 & 1332 & 1060 & 1060 & 1060 & 1060 & 1060 & 1060 & 1060 \\
\hline surface (square meter) & 16 & 16 & 16 & 25 & 4 & 16 & 36 & 20 & 16 & 16 \\
\hline Cover (\%) & 100 & 95 & 95 & 100 & 100 & 100 & 100 & 100 & 100 & 100 \\
\hline Author & Venanzoni & $\begin{array}{c}\text { Ven. \& } \\
\text { Pralesk. }\end{array}$ & Venanzoni & Venanzoni & Venanzoni & Venanzoni & Venanzoni & Venanzoni & Venanzoni & Venanzoni \\
\hline
\end{tabular}

